\title{
Generic Hopf bifurcation from lines of equilibria without parameters:
}

\section{Systems of viscous hyperbolic balance laws *}

\author{
Bernold Fiedler, Stefan Liebscher
}

\author{
Institut für Mathematik I \\ Freie Universität Berlin \\ Arnimallee 2-6, 14195 Berlin, Germany
}

June 2, 1998

*This work was supported by the DFG-Schwerpunkt "Analysis und Numerik von Erhaltungsgleichungen" and with funds provided by the "National Science Foundation" at IMA, University of Minnesota, Minneapolis. 


\section{Introduction}

Searching for viscous shock profiles of the Riemann problem, we consider systems of hyperbolic balance laws of the form

$$
u_{t}+f(u)_{x}=\epsilon^{-1} g(u)+\epsilon \delta u_{x x}
$$

with $u=\left(u_{0}, u_{1}, \cdots, u_{N}\right) \in \mathbb{R}^{N+1}, f \in C^{3}, g \in C^{2}, \delta>0$, and with real time $t$ and space $x$. We assume strict hyperbolicity, that is, the Jacobian $A(u)=f^{\prime}(u)$ possesses simple real distinct eigenvalues

$$
\sigma_{0}, \sigma_{1}, \cdots, \sigma_{N} \in \operatorname{spec} A(u)
$$

The case of conservation laws, $g \equiv 0$, has been studied extensively. See for example [Smo83] for a background. Viscous profiles are traveling wave solutions of the form

$$
u=u\left(\frac{x-s t}{\epsilon}\right)
$$

with wave speed $s$. Here (1.3) provides a solution of (1.1) if

$$
-s \dot{u}+A(u) \dot{u}=g(u)+\delta \ddot{u}
$$

Here $A(u)=f^{\prime}(u)$ denotes the Jacobian and $=\frac{d}{d \tau}$ with $\tau=(x-s t) / \epsilon$. Note that (1.4) is independent of $\epsilon>0$. Any solution of system (1.4) for which

$$
\lim _{\tau \rightarrow \pm \infty} u(\tau)=u^{ \pm}
$$

exist gives rise, for $\epsilon \searrow 0$, to a solution of the Riemann problem of (1.1) with values $u=u^{ \pm}$connected by a shock traveling with shock speed $s$. We call solutions $u(\cdot)$ of $(1.4),(1.5)$ viscous profiles.

We rewrite the viscous profile equation (1.4) as a second order system

$$
\begin{aligned}
\dot{u} & =v \\
\delta \dot{v} & =-g(u)+(A(u)-s) v
\end{aligned}
$$


Note that any viscous profile must satisfy

$$
g\left(u^{ \pm}\right)=0
$$

In other words, the asymptotic states $u^{ \pm}$must be equilibria of the reaction term $g(u)$. In the conservation law case, $g \equiv 0$, this condition does not impose any restraint on the Riemann values $u^{ \pm}$. In the other extreme of a reaction term $g$ with unique equilibrium, we obtain $u^{+}=u^{-}$and traveling shock profiles do not exist. In the present paper, we assume that the $u_{0}-$ component does not contribute to the reaction terms and still all reaction components vanish, say at $u=0$. Specifically, we assume throughout this paper that

$$
g=g\left(u_{1}, \cdots, u_{N}\right)=\left(\begin{array}{c}
g_{0} \\
g_{1} \\
\vdots \\
g_{N}
\end{array}\right)
$$

is independent of $u_{0}$ and satisfies

$$
g(0)=0 .
$$

This gives rise to a line of equilibria

$$
u_{0} \in \mathbb{R}, \quad u_{1}=\cdots=u_{N}=0, \quad v=0
$$

of our viscous profile system (1.6).

The asymptotic behavior of viscous profiles $u(\tau)$ for $\tau \rightarrow \pm \infty$ depends on the linearization $L$ of $(1.6)$ at $u=u_{ \pm}, v=0$. In block matrix notation corresponding to coordinates $(u, v)$ we have

$$
L=\left(\begin{array}{cc}
0 & \text { id } \\
-\delta^{-1} g^{\prime} & \delta^{-1}(A-s)
\end{array}\right)
$$


Here $A=A(u)$, and $g^{\prime}=g^{\prime}(u)$ describes the Jacobi matrix of the reaction term $g$ at $u=u^{ \pm}$. In (1.11) we write $s$ rather than $s \cdot$ id, for brevity. In the case $g \equiv 0$ of pure conservation laws, the linearization $L$ possesses an $(N+1)$-dimensional kernel corresponding to the then arbitrary choice of the equilibrium $u \in \mathbb{R}^{N+1}, v=0$. Normal hyperbolicity of this family of equilibria, in the sense of dynamical systems [HPS77], [Fen77], [Wig94], is ensured for wave speeds $s$ not in the spectrum of the strictly hyperbolic Jacobian $A(u)$ :

$$
s \notin \operatorname{spec} A(u)=\left\{\sigma_{0}, \cdots, \sigma_{N}\right\} .
$$

Indeed, (1.12) ensures that additional zeros do note arise in the real spectrum

$$
\operatorname{spec} L=\{0\} \cup \delta^{-1} \operatorname{spec}(A(u)-s)
$$

In the present paper we investigate the failure of normal hyperbolicity of $L$ along the line of equilibria $u=\left(u_{0}, 0, \cdots, 0\right), v=0$ given by (1.10). Although our method applies in complete generality, we just present a simple specific example for which purely imaginary eigenvalues of $L$ arise when $\delta>0$ is fixed small enough. Specifically, we consider three-dimensional systems, $N=2$, satisfying

$$
\begin{aligned}
A\left(u_{0}, 0,0\right) & =A_{0}+u_{0} \cdot A_{1} \\
A_{0} & =\left(\begin{array}{rrr}
\alpha & & \\
& 1 & \\
& & -1
\end{array}\right), \quad \alpha \neq 0, A_{1} \text { symmetric } \\
g^{\prime}(0) & =\left(\begin{array}{rrr}
0 & & \\
& \gamma & 1 \\
& 1 & \gamma
\end{array}\right), \quad|\gamma|<1,
\end{aligned}
$$


with omitted entries being zero. Note that these data can arise from flux functions $f$ which are gradient vector fields, still giving rise to purely imaginary eigenvalues. At the end of this paper we present a specific example where the reaction terms $\dot{u}=g(u)$ alone, likewise, do not support even transient oscillatory behavior; see (3.12). The interaction of flux and reaction, in contrast, is able to produce purely imaginary eigenvalues of the linearization $L$ as follows.

\section{Proposition 1.1}

Consider the linearization $L=L\left(u_{0}\right)$ along the line $u_{0} \in \mathbb{R}, u_{1}=u_{2}=0$ of equilibria of the viscous profile system (1.6) in $\mathbb{R}^{3}$; see (1.11). Assume (1.14) holds.

For $\delta \searrow 0$, and small $|s|,\left|u_{0}\right|$, the spectrum of $L$ then decouples into two parts:

(i) an unboundedly growing part $\operatorname{spec}_{\infty}(L)=\delta^{-1} \operatorname{spec}(A-s)+O(1)$

(ii) a bounded part $\operatorname{spec}_{b d}(L)=\operatorname{spec}\left((A-s)^{-1} g^{\prime}\right)+O(\delta)$

Here $A, g$ are evaluated at $u=\left(u_{0}, 0,0\right)$.

For $\delta=0, s=0,|\gamma|<1$, the bounded part $\operatorname{spec}_{b d}(L)$ at $u_{0}=0$ limits onto simple eigenvalues $\mu_{0} \in\left\{0, \pm i \omega_{0}\right\}, \omega_{0}=\sqrt{1-\gamma^{2}}$ with eigenvectors $\left(\begin{array}{c}\tilde{u} \\ \tilde{v}\end{array}\right)$ given by $\tilde{v}=\mu_{0} \tilde{u}$ and

$$
\begin{aligned}
\tilde{u}=\left(\begin{array}{l}
1 \\
0 \\
0
\end{array}\right), & \text { for } \mu_{0}=0 \\
\tilde{u}=\left(\begin{array}{c}
0 \\
-\gamma-i \omega_{0} \\
1
\end{array}\right), & \text { for } \mu_{0}=+i \omega_{0} .
\end{aligned}
$$




\section{Proof:}

Regular perturbation theory applies to the scaled block matrix

$$
\delta L=\left(\begin{array}{rl}
0 & 0 \\
-g^{\prime} & A-s
\end{array}\right)+\delta\left(\begin{array}{ll}
0 & \text { id } \\
0 & 0
\end{array}\right)
$$

which becomes lower triangular for $\delta=0$. This provides us with the unbounded part $\operatorname{spec}_{\infty}(L)$ of the spectrum, generated in $v$-space alone with $u=0$.

Moreover $\delta L$ possesses three-dimensional kernel, at $\delta=0$, given by

$$
g^{\prime} u=(A-s) v
$$

On this kernel, the eigenvalue problem for $L$ reduces to

$$
\mu_{0} u=v=(A-s)^{-1} g^{\prime} u
$$

The characteristic polynomial of (1.18) at $u_{0}=0$ is given by

$$
p_{0}(\mu)=\left(\mu^{2}-\frac{2 \gamma s}{1-s^{2}} \mu+\frac{1-\gamma^{2}}{1-s^{2}}\right) \mu
$$

Direct calculation completes the proof.

For explicit calculations here and below, we have used and recommend assistance by symbolic packages like Mathematica, Maple, etc.

Bifurcations from lines of equilibria in absence of parameters have been investigated in [Lie97], [FLA98a] from a theoretical view point. We briefly recall that result, for convenience. Consider $C^{2}$ vector fields

$$
\dot{\mathbf{u}}=F(\mathbf{u})
$$

with $\mathbf{u}=\left(u_{0}, u_{1}, \cdots, u_{n}\right) \in \mathbb{R}^{n+1}$. We assume a line of equilibria

$$
0=F\left(u_{0}, 0, \cdots, 0\right)
$$


along the $u_{0}$-axis. At $u_{0}=0$, we assume the Jacobi matrix $F^{\prime}\left(u_{0}, 0, \cdots, 0\right)$ to be hyperbolic, except for a trivial kernel vector along the $u_{0}$-axis and a complex conjugate pair of simple, purely imaginary, nonzero eigenvalues $\mu\left(u_{0}\right), \overline{\mu\left(u_{0}\right)}$ crossing the imaginary axis transversely as $u_{0}$ increases through $u_{0}=0$ :

$$
\begin{aligned}
\mu(0) & =i \omega(0), \quad \omega(0)>0 \\
\operatorname{Re} \mu^{\prime}(0) & \neq 0
\end{aligned}
$$

Let $Z$ be the two-dimensional real eigenspace of $F^{\prime}(0)$ associated to $\pm i \omega(0)$. By $\Delta_{Z}$ we denote the Laplacian with respect to variations of $u$ in the eigenspace $Z$. Coordinates in $Z$ are chosen as coefficients of the real and imaginary parts of the complex eigenvector associated to $i \omega(0)$. Note that the linearization acts as a rotation with respect to these not necessarily orthogonal coorrdinates. Let $P_{0}$ be the one-dimensional eigenprojection onto the trivial kernel along the $u_{0}$-axis. Our final nondegeneracy assumption then reads

$$
\Delta_{Z} P_{0} F(0) \neq 0
$$

Fixing orientation along the positive $u_{0}$-axis, we can consider $\Delta_{Z} P_{0} F(0)$ as a real number. Depending on the sign

$$
\eta:=\operatorname{sign}\left(\operatorname{Re} \mu^{\prime}(0)\right) \cdot \operatorname{sign}\left(\Delta_{Z} P_{0} F(0)\right),
$$

we call the "bifurcation" point $u_{0}=0$ elliptic, if $\eta=-1$, and hyperbolic for $\eta=+1$.

The following result from [FLA98a] investigates the qualitative behavior of solutions in a normally hyperbolic three-dimensional center manifold to $\mathbf{u}=$ 0 .

The results for the hyperbolic case $\eta=+1$ are based on normal form theory and a spherical blow-up construction inside the center manifold. The elliptic case $\eta=-1$ is based on Neishtadt's theorem on exponential elimination of 
rapidly rotating phases [Nei84]. For a related application to binary oscillators in discretized systems of hyperbolic balance laws see [FLA98b]. For an application to square rings of additively coupled oscillators see [AF98].

\section{Theorem 1.2}

Let assumptions (1.21) - (1.23) hold for the $C^{5}$ vector field $\dot{\mathbf{u}}=F(\mathbf{u})$. Then the following holds true in a neighborhood $U$ of $\mathbf{u}=0$ within a threedimensional center manifold to $\mathbf{u}=0$.

In the hyperbolic case, $\eta=+1$, all nonequilibrium trajectories leave the neighborhood $U$ in positive or negative time direction (possibly both). The stable and unstable sets of $\mathbf{u}=0$, respectively, form cones around the positive/negative $u_{0}$-axis, with asymptotically elliptic cross section near their tips at $\mathbf{u}=0$. These cones separate regions with different convergence behavior. See Fig. 1.1, a).

In the elliptic case all nonequilibrium trajectories starting in $U$ are heteroclinic between equilibria $\mathbf{u}^{ \pm}=\left(u_{0}^{ \pm}, 0, \cdots, 0\right)$ on opposite sides of $u_{0}=0$. If $F(\mathbf{u})$ is real analytic near $\mathbf{u}=0$, then the two-dimensional strong stable and strong unstable manifolds of $\mathbf{u}^{ \pm}$within the center manifold intersect at an angle which possesses an exponentially small upper bound in terms of $\left|\mathbf{u}^{ \pm}\right|$. See Fig. 1.1,b).

In the present paper, we apply theorem 1.2 to the problem of zero speed viscous profiles of systems of hyperbolic balance laws near Hopf points as in proposition 1.1. Nonzero shock speeds can be treated completely analogously, absorbing them into the flux term.

\section{Theorem 1.3}

Consider the problem (1.5)-(1.7) of finding viscous profiles with shock speed $s=0$ to hyperbolic balance laws (1.1). Let assumptions (1.8)-(1.10), (1.14) 


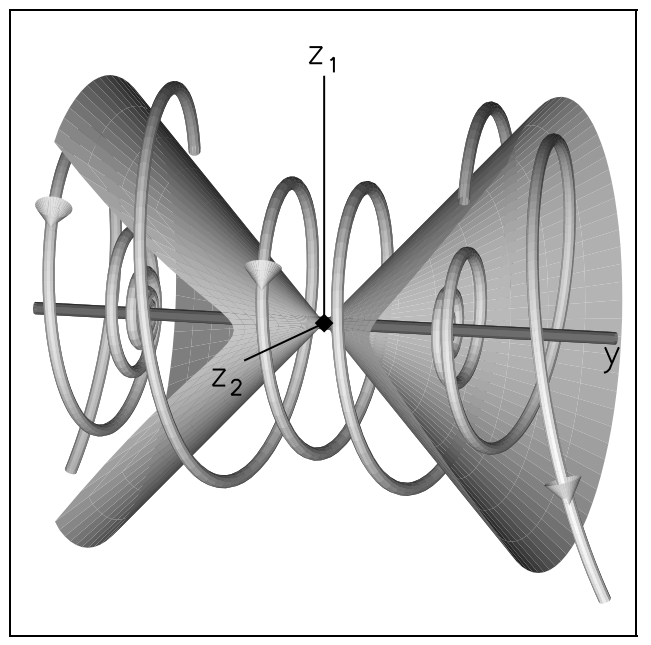

Case a) hyperbolic, $\eta=+1$.

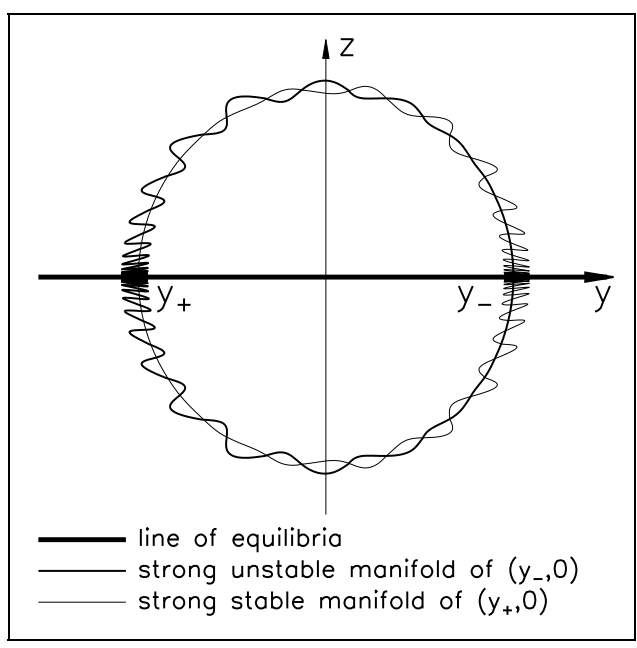

Case b) elliptic, $\eta=-1$.

Figure 1.1: Dynamics near Hopf bifurcation from lines of equilibria.

hold, so that a pair of purely imaginary simple eigenvalues occurs for the linearization $L$, in the limit $\delta \rightarrow 0$.

Then there exist nonlinearities $A(u)=f^{\prime}(u)$ and $g(u)$, compatible with the above assumptions, such that the assumptions and conclusions of theorem 1.2 are valid for the viscous profile system (1.6). Both the elliptic and the hyperbolic case occur; see fig. 1.1.

Since both conditions are open, the results persist in particular for small nonzero shock speeds $s$, even when $f, g$ remain fixed.

Specific choices of flux $f(u)$ and reaction terms $g(u)$ are presented in corollary 3.3; see (3.12). In the elliptic case $\eta=-1$, we nevertheless observe (at least) pairs of weak shocks with oscillatory tails, connecting $u_{-}$and $u_{+}$. In the hyperbolic case $\eta=+1$, viscous profiles leave the neighborhood $U$ and thus represent large shocks. At $u_{0}=0$, their profiles change discontinuously and the role of the $u_{0}$-axis switches from left to right asymptotic state with oscillatory tail. 
This paper is organized as follows. In section 2 we check transversality condition (1.22) for the purely imaginary eigenvalues. We also compute an expansion in terms of $\delta$ for the eigenprojection $P_{0}$ onto the trivial kernel along the $u_{0}$-axis. In section 3 , we check nondegeneracy condition (1.23) for $\Delta_{Z} P_{0} F(0)$, in the limit $\delta \searrow 0$, completing the proof of theorem 1.3 by reduction to theorem 1.2 .

\section{Acknowledgment}

Helpful discussions with Bob Pego and Heinrich Freistühler concerning the set-up of viscous profiles for hyperbolic balance laws are gratefully acknowledged. This work was supported by the Deutsche Forschungsgemeinschaft, Schwerpunkt "Analysis und Numerik von Erhaltungsgleichungen" and was completed during a fruitful and enjoyable research visit of one of the authors at the Institute of Mathematics and its Applications, University of Minnesota, Minneapolis. This research was supported in part by the Institute for Mathematics and its Applications with funds provided by the National Science Foundation.

\section{Linearization and transverse eigenvalue crossing}

In this section we continue our linear analysis of the linearization

$$
L^{\delta}\left(u_{0}\right)=\left(\begin{array}{cc}
0 & \mathrm{id} \\
-\delta^{-1} g^{\prime} & \delta^{-1} A
\end{array}\right)
$$

with $A=A(u), g^{\prime}=g^{\prime}(u)$ evaluated along the line of equilibria $u=\left(u_{0}, 0,0\right)$. See (1.11) with $s=0$ and proposition 1.1. In the limit $\delta \searrow 0$, we address 
the issue of transverse crossing of purely imaginary eigenvalues in lemma 2.1. In lemma 2.2, we explicitly compute the one-dimensional eigenprojection $P_{0}^{\delta}$ onto the trivial kernel of $L^{\delta}(0)$.

Throughout this section we fix the notation

$$
A\left(u_{0}, 0,0\right)=A_{0}+u_{0} A_{1}=\left(\begin{array}{ccc}
\alpha & & \\
& 1 & \\
& & -1
\end{array}\right)+u_{0} \cdot\left(a_{j k}^{1}\right)_{0 \leq j, k \leq 2}
$$

with $a_{j k}^{1}=a_{k j}^{1}$ symmetric; see (1.14). We also assume that the linearized reaction term

$$
g^{\prime}\left(u_{0}, 0,0\right)=g^{\prime}(0)=\left(\begin{array}{ccc}
0 & & \\
& \gamma & 1 \\
& 1 & \gamma
\end{array}\right), \quad|\gamma|<1
$$

which is independent of $u_{0}$ by assumption (1.8), possesses a vanishing $g_{0}-$ component.

By proposition 1.1, purely imaginary eigenvalues of $L^{\delta}\left(u_{0}\right)$ arise from an $O(\delta)$ perturbation of the matrix

$$
A^{-1} g^{\prime}=\left(A_{0}+u_{0} A_{1}\right)^{-1} g^{\prime}(0)
$$

with spectrum $\operatorname{spec}_{b d}(L)$. Let

$$
\mu\left(u_{0}\right), \quad \bar{\mu}\left(u_{0}\right)
$$

denote the continuation of the simple, purely imaginary eigenvalues

$$
\mu(0)=i \omega_{0}, \quad \bar{\mu}(0)=-i \omega_{0}
$$

with $u_{0}$-derivatives $\mu^{\prime}\left(u_{0}\right), \bar{\mu}^{\prime}\left(u_{0}\right)$. 


\section{Lemma 2.1}

In the above setting and notation,

$$
\operatorname{Re} \mu^{\prime}(0)=-\frac{\gamma}{2}\left(a_{11}^{1}+a_{22}^{1}\right)+a_{12}^{1} .
$$

\section{Proof :}

Since the unit vector $e_{0}$ in $u_{0}$-direction is a trivial kernel vector of $g^{\prime}(0)$ and since the remaining eigenvalues of $A^{-1} g^{\prime}$ remain conjugate complex for small $\left|u_{0}\right|$, we have

$$
\operatorname{Re} \mu\left(u_{0}\right)=\frac{1}{2} \operatorname{trace}\left(A^{-1} g^{\prime}\right) .
$$

In particular, trace $A_{0}^{-1} g^{\prime}=0$. With the $u_{0}$-expansion

$$
A^{-1}=\left(A_{0}+u_{0} A_{1}\right)^{-1}=A_{0}^{-1}-u_{0} A_{0}^{-1} A_{1} A_{0}^{-1}+\cdots
$$

we immediately obtain

$$
\operatorname{Re} \mu^{\prime}\left(u_{0}\right)=-\frac{1}{2} \operatorname{trace}\left(A_{0}^{-1} A_{1} A_{0}^{-1} g^{\prime}\right)
$$

Inserting $A_{0}, A_{1}, g^{\prime}$ proves the lemma.

By regular perturbation of $\operatorname{spec}_{b d}(L)$, the result $\operatorname{Re} \mu^{\prime}(0) \neq 0$ of lemma 2.1 extends to small positive $\delta$.

We now turn to an expansion for the eigenprojection $P_{0}^{\delta}$ onto the onedimensional kernel of the $6 \times 6$-matrix $L^{\delta}\left(u_{0}\right)$ at $u_{0}=0$; see $(2.1),(2.2)$. Aligning the notations of proposition 1.1 and of theorem 1.2, we decompose

$$
\mathbf{u}=(u, v) \in \mathbb{R}^{6}=\mathbb{R}^{3} \times \mathbb{R}^{3} .
$$

Again, $e_{0}^{T}=(1,0,0)$ denotes the first unit vector in $\mathbb{R}^{3}$ and $\mathbf{e}_{0}^{T}=\left(e_{0}^{T}, 0\right)$ the first unit vector in $\mathbb{R}^{6}$. 


\section{Lemma 2.2}

In the above setting and notation

$$
\begin{aligned}
& P_{0}^{\delta}=\mathbf{e}_{0} \cdot \mathbf{e}_{\delta}^{T}, \quad \text { with } \\
& \mathbf{e}_{\delta}^{T}=\left(1+\left(\frac{\delta}{\alpha}\right)^{2}\right)^{-1 / 2}\left(e_{0}^{T},-\frac{\delta}{\alpha} e_{0}^{T}\right)
\end{aligned}
$$

\section{Proof :}

Kernel and co-kernel of $L^{\delta}\left(u_{0}\right)$ are one-dimensional, corresponding to the simple zero eigenvalue of $L^{\delta}\left(u_{0}\right)$. Obviously

$$
\operatorname{ker} L^{\delta}\left(u_{0}\right)=\mathbf{e}_{0}
$$

because $g^{\prime}\left(u_{0}, 0,0\right) e_{0}=0$. At $u_{0}=0$, the co-kernel of $L^{\delta}\left(u_{0}\right)$ is given by

$$
0=\mathbf{e}_{\delta}^{T} \cdot\left(\begin{array}{cc}
0 & \mathrm{id} \\
-\delta^{-1} g^{\prime} & \delta^{-1} A_{0}
\end{array}\right)
$$

Inserting $A_{0}$ from (1.14) proves the lemma.

\section{$3 \quad$ Higher order nondegeneracy}

In this section we complete the proof of theorem 1.3. In view of theorem 1.2, we have already checked transverse crossing of purely imaginary eigenvalues, assumption (1.22), in lemma 2.1. Letting

$$
F(\mathbf{u})=F(u, v)=\left(\begin{array}{c}
v \\
-\delta^{-1} g(u)+\delta^{-1} A(u) v
\end{array}\right)
$$

it remains to check the nondegeneracy assumption $\Delta_{Z} P_{0} F \neq 0$; see (1.23). In lemma 3.1, we check this assumption in the limit $\delta \searrow 0$. In corollary 3.2, we provide explicit expressions for the type determining sign $\eta= \pm 1$ defined in (1.24). In particular, we show in corollary 3.3 that both the hyperbolic 
case $\eta=+1$ and the elliptic case $\eta=-1$ can be realized by our nonlinear hyperbolic balance laws, even with gradient flux terms. This then completes the proof of theorem 1.3.

To check nondegeneracy condition (1.23) on $\Delta_{Z} P_{0} F$ in the limit $\delta \searrow 0$, we use the following notation. By transverse eigenvalue crossing at $\delta=0$, lemma 2.1 , we also obtain purely imaginary eigenvalues $\pm i \omega^{\delta}$ at equilibria $\mathbf{u}^{\delta}=$ $\left(u_{0}^{\delta}, 0, \cdots, 0\right)=\left(u^{\delta}, 0\right)$ on the $u_{0}$-axis, for small $\delta>0$. Let $Z^{\delta}$ denote the corresponding eigenspace. We recall our expression for the eigenprojection $P_{0}^{\delta}$ onto the trivial kernel,

$$
P_{0}^{\delta}=\left(1+\left(\frac{\delta}{\alpha}\right)^{2}\right)^{-1 / 2} \mathbf{e}_{0} \cdot\left(e_{0}^{T},-\frac{\delta}{\alpha} e_{0}^{T}\right)
$$

with $\mathbf{e}_{0}=\left(e_{0}^{T}, 0\right)^{T}$, see lemma 2.2. Note that $\mathbf{u}^{\delta}, \omega^{\delta}, P_{0}^{\delta}$, and $Z^{\delta}$ vary differentiably with $\delta$.

\section{Lemma 3.1}

In the above setting and notation, we have

$$
\Delta_{Z^{\delta}} P_{0}^{\delta} F\left(\mathbf{u}^{\delta}\right)=\frac{1}{\alpha} g_{0}^{\prime \prime}\left(u^{\delta}\right)\left[\tilde{u}^{\delta}, \overline{\hat{u}}^{\delta}\right]
$$

at the Hopf point $\mathbf{u}^{\delta}=\left(u^{\delta}, 0\right)$ with complex eigenvector $\left(\tilde{u}^{\delta}, \tilde{v}^{\delta}\right)$ of $i \omega^{\delta}$.

Consider in particular quadratic forms $g_{0}^{\prime \prime}(0)$, which are strictly positive/negative definite on $\left(u_{1}, u_{2}\right)$-space, with $\Gamma= \pm 1$ indicating the sign of definiteness. Then

$$
\operatorname{sign} \Delta_{Z^{\delta}} P_{0}^{\delta} F\left(\mathbf{u}^{\delta}\right)=\Gamma \cdot \operatorname{sign} \alpha
$$

for all small $\delta>0$.

\section{Proof :}

By lemma 2.2 we have

$$
\left(1+\left(\frac{\delta}{\alpha}\right)^{2}\right)^{1 / 2} P_{0}^{\delta}=\mathbf{e}_{0} \cdot \mathbf{e}_{0}^{T}-\frac{\delta}{\alpha} \mathbf{e}_{0} \cdot\left(0, e_{0}^{T}\right)
$$


The explicit form (3.1) of the nonlinearity $F$ implies

$$
\Delta_{Z^{\delta}} P_{0}^{0} F\left(\mathbf{u}^{\delta}\right)=\mathbf{e}_{0} \Delta_{Z^{\delta}} v_{0}=0
$$

on any subspace $Z^{\delta}$ and for any $\mathbf{u}^{\delta}$, simply because the $u$-component of $F$ is linear. With $P_{0}^{\delta}$ instead of $P_{0}^{0}$ we obtain more generally

$$
\begin{aligned}
\left(1+\left(\frac{\delta}{\alpha}\right)^{2}\right)^{1 / 2} \mathbf{e}_{0}^{T} \Delta_{Z^{\delta}} P_{0}^{\delta} F(\mathbf{u}) & =-\Delta_{Z^{\delta}}\left(\left(0,-\frac{\delta}{\alpha} e_{0}^{T}\right)\left(\delta^{-1}(-g(u)+A(u) v)\right)\right) \\
& =-\frac{1}{\alpha} \Delta_{Z^{\delta}}\left(-g_{0}(u)+(A(u) v)_{0}\right) .
\end{aligned}
$$

Here $(A(u) v)_{0}$ denotes the zero-component of $A(u) v$. We treat this term first, using the notation

$$
\tilde{\mathbf{u}}^{\delta}=\left(\begin{array}{c}
\tilde{u}^{\delta} \\
\tilde{v}^{\delta}
\end{array}\right)
$$

for the complex eigenvector of the purely imaginary Hopf eigenvalue $\mu^{\delta}=i \omega^{\delta}$ at $u=u^{\delta}, v=0$. Then $Z^{\delta}=\operatorname{span}\left\{\operatorname{Re} \tilde{\mathbf{u}}^{\delta}, \operatorname{Im} \tilde{\mathbf{u}}^{\delta}\right\}$. Denoting by $\Delta_{\beta}=\partial_{\beta_{1}}^{2}+\partial_{\beta_{2}}^{2}$ the standard Laplacian, evaluated at $\beta=0$, and inserting $\tilde{v}^{\delta}=\mu^{\delta} \tilde{u}^{\delta}$ yields

$$
\begin{aligned}
\Delta_{Z^{\delta}}(A(u) v)_{0} & =\Delta_{\beta}\left(A\left(u^{\delta}+\beta_{1} \operatorname{Re} \tilde{u}^{\delta}+\beta_{2} \operatorname{Im} \tilde{u}^{\delta}\right)\left(\beta_{1} \operatorname{Re} \tilde{v}^{\delta}+\beta_{2} \operatorname{Im} \tilde{v}^{\delta}\right)\right)_{0} \\
& =2\left(\left(A^{\prime}\left(u^{\delta}\right) \operatorname{Re} \tilde{u}^{\delta}\right) \operatorname{Re} \tilde{v}^{\delta}+\left(\left(A^{\prime}\left(u^{\delta}\right) \operatorname{Im} \tilde{u}^{\delta}\right) \operatorname{Im} \tilde{v}^{\delta}\right)_{0}=\right. \\
& =2 \operatorname{Re}\left(\left(A^{\prime}\left(u^{\delta}\right) \tilde{u}^{\delta}\right) \bar{v}^{\delta}\right)_{0}= \\
& =2 \operatorname{Re}\left(\bar{\mu}^{\delta}\right)\left(f^{\prime \prime}\left(u^{\delta}\right)\left[\tilde{u}^{\delta}, \overline{\tilde{u}}^{\delta}\right]\right)_{0}= \\
& =2 \operatorname{Re}\left(\mu^{\delta}\right) f_{0}^{\prime \prime}\left(u^{\delta}\right)\left[\tilde{u}^{\delta}, \overline{\tilde{u}}^{\delta}\right]=0
\end{aligned}
$$

all along the Hopf curve $u=u^{\delta}, v=0$. Here we have used $A(u)=f^{\prime}(u)$ for the flux function and the fact that the Hessian matrix $f_{0}^{\prime \prime}(0)$ is symmetric. Therefore, we can conclude from (3.7), (3.9) that

$$
\Delta_{Z^{\delta}} P_{0}^{\delta} F\left(\mathbf{u}^{\delta}\right)=\frac{1}{\alpha} \Delta_{Z^{\delta}} g_{0}(u)=\frac{1}{\alpha} g_{0}^{\prime \prime}\left(u^{\delta}\right)\left[\tilde{u}^{\delta}, \overline{\tilde{u}}^{\delta}\right]
$$

This proves (3.3), and the lemma. 


\section{Corollary 3.2}

Combining lemmata 2.1 and 3.1, the sign $\eta= \pm 1$ distinguishing elliptic from hyperbolic Hopf bifurcation along our line of equilibria is given explicitly by

$$
\begin{aligned}
\eta & =\operatorname{sign} \operatorname{Re} \mu^{\prime}(0) \cdot \operatorname{sign} \Delta_{Z} P_{0} F(0) \\
& =\operatorname{sign}\left(\partial_{u_{0}} a_{12}-\frac{\gamma}{2} \partial_{u_{0}}\left(a_{11}+a_{22}\right)\right) \cdot \operatorname{sign} \alpha \cdot \Gamma
\end{aligned}
$$

for $\delta>0$ small enough. Here derivatives are evaluated at $u=0$, and are assumed to be chosen such that $\eta \neq 0$. The sign $\Gamma= \pm 1$ indicates positive/negative definiteness of $g_{0}^{\prime \prime}(0)$ on $\left(u_{1}, u_{2}\right)$-space. Obviously, both signs of $\eta$ can be realized.

\section{Corollary 3.3}

Theorems 1.2, 1.3 hold true for $\eta= \pm 1$ with the following specific choices of a gradient flux term $f(u)=\nabla \Phi(u)$ and a reaction term $g(u)$ :

$$
\begin{aligned}
g(u) & =\left(\begin{array}{ccc}
u_{1}^{2} & + & u_{2}^{2} \\
\frac{1}{2} u_{1} & + & u_{2} \\
u_{1} & + & \frac{1}{2} u_{2}
\end{array}\right) \\
\Phi(u) & =u_{0}^{2}+\frac{1}{2}\left(u_{1}^{2}-u_{2}^{2}\right)+\frac{1}{2} \eta u_{0} u_{1}^{2}+u_{0} u_{2}^{2}
\end{aligned}
$$

These choices correspond to $\alpha=2, \gamma=\frac{1}{2}, \Gamma=+1$.

\section{References}

[AF98] J.C. Alexander and B. Fiedler. Stable and unstable decoupling in squares of additively coupled oscillators. In preparation, 1998.

[Fen77] N. Fenichel. Asymptotic stability with rate conditions, II. Indiana Univ. Math. J., 26:81-93, (1977). 
[FLA98a] B. Fiedler, S. Liebscher, and J.C. Alexander. Generic Hopf bifurcation from lines of equilibria without parameters: I. Theory. Preprint, FU Berlin, 1998.

[FLA98b] B. Fiedler, S. Liebscher, and J.C. Alexander. Generic Hopf bifurcation from lines of equilibria without parameters: III. Binary oscillations. Preprint, FU Berlin, 1998.

[HPS77] M.W. Hirsch, C.C. Pugh, and M. Shub. Invariant Manifolds. Lect. Notes Math. 583. Springer-Verlag, Berlin, 1977.

[Lie97] S. Liebscher. Stabilität von Entkopplungsphänomenen in Systemen gekoppelter symmetrischer Oszillatoren. Diploma Thesis, 1997.

[Nei84] A. Neishtadt. On the seperation of motions in systems with rapidly rotating phase. J. Appl. Math. Mech., 48:134-139, (1984).

[Smo83] J. Smoller. Shock Waves and Reaction-Diffusion Equations. Springer-Verlag, New York, 1983.

[Wig94] S. Wiggins. Normally Hyperbolic Invariant Manifolds in Dynamical Systems. Springer-Verlag, New York, 1994. 